\title{
Destruction of formic acid by soft X-rays in star-forming regions
}

\author{
H. M. Boechat-Roberty ${ }^{1}$, S. Pilling ${ }^{1,2}$, and A. C. F. Santos ${ }^{3}$ \\ 1 Observatório do Valongo, Universidade Federal do Rio de Janeiro, Ladeira Pedro Antônio 43, CEP 20080-090, Rio de Janeiro, \\ Brazil \\ e-mail: heloisa@ov.ufrj.br \\ 2 Instituto de Química, Universidade Federal do Rio de Janeiro, Ilha do Fundão, CEP 21949-900, Rio de Janeiro, Brazil \\ 3 Instituto de Física, Universidade Federal do Rio de Janeiro, Caixa Postal 68528, CEP 21941-972, Rio de Janeiro, Brazil
}

Received 21 December 2004 / Accepted 1 April 2005

\begin{abstract}
Formic acid is much more abundant in the solid state, both in interstellar ices and cometary ices, than in the interstellar gas (ice/gas $\sim 10^{4}$ ) and this point remains a puzzle. The goal of this work is to experimentally study ionization and photodissociation processes of $\mathrm{HCOOH}$ (formic acid), a glycine precursor molecule. The measurements were taken at the Brazilian Synchrotron Light Laboratory (LNLS), employing soft X-ray photons from toroidal grating monochromator TGM) beamline (200-310 eV). Mass spectra were obtained using photoelectron photoion coincidence (PEPICO) method. Kinetic energy distributions and abundances for each ionic fragment have been obtained from the analysis of the corresponding peak shapes in the mass spectra. Photoionization and photodissociation cross sections were also determined. Due to the large photodissociation cross section of $\mathrm{HCOOH}$ it is possible that in PDRs regions, just after molecules evaporation from the grain surface, formic acid molecules are almost totally destroyed by soft X-rays, justifying the observed low abundance of $\mathrm{HCOOH}$ in the gaseous phase. The preferential path for the glycine formation from formic acid may be through the ice phase reaction.
\end{abstract}

Key words. astrochemistry - molecular processes - ISM: abundances - ISM: molecules - X-rays: ISM - astrobiology

\section{Introduction}

Formic acid (HCOOH) has been observed in various astronomical sources: protostellar ices NGC 7538:IRS9 (Ehrenfreund \& Shutte 2000), comet Hale-Bopp (Crovisier \& Bockelée-Morvan 1999), dark molecular clouds (Ohshi et al. 1992) and Photodissociation Regions (PDRs) associated with Hot Molecular Cores (Zuckermann et al. 1971; Liu et al. 2001; Liu et al. 2002). Cazaux et al. (2003) observed an extremely rich organic inventory in the hot core around the low-mass protostar IRAS 16293-24 with abundant amounts of molecules such as formic acid, acetaldehyde $\left(\mathrm{CH}_{3} \mathrm{CHO}\right)$, methyl formate $\left(\mathrm{CH}_{3} \mathrm{OCHO}\right)$, dimethyl ether $\left(\mathrm{CH}_{3} \mathrm{OCH}_{3}\right)$ and acetic acid $\left(\mathrm{CH}_{3} \mathrm{COOH}\right)$. Bottinelli et al. (2004) also reported the detection of formic acid in the hot core of the low mass protostar NGC 133-IRAS4A. It is known that protostars are sources of X-rays (Koyama et al. 1996).

The simplest amino acid, glycine, $\left(\mathrm{NH}_{2} \mathrm{CH}_{2} \mathrm{COOH}\right)$, was recently detected in the molecular clouds SgrB2, Orion $\mathrm{KL}$ and W51 (Kuan et al. 2003, 2004). In these objects the precursor molecules like ammonia, formic acid and acetic acid have already been observed (Turner 1991; Sutton et al. 1985). Liu et al. (2002) pointed out the importance of performing studies on formic acid since it is the simplest organic molecule and shares common structural elements with biologically important species such as acetic acid and glycine.
Sgr B2, Orion KL and W51 are massive star-forming regions where the presence of widespread UV and X-ray fields could trigger the formation of photodissociation regions (PDRs). X-ray photons are capable of traversing large column densities of gas before being absorbed. X-ray-dominated regions (XDRs) in the interface between the ionized gas and the self-shielded neutral layers could influence the selective heating of the molecular gas. The complexity of the region possibly allows a combination of different scenarios and excitation mechanisms to coexist within the complex (Goicoechea et al. 2004).

The formation of $\mathrm{HCOOH}$ in molecular clouds by gasphase reactions has been suggested by Irvine et al. (1990). On the other hand, experiments and models of grain-surface chemistry suggest that $\mathrm{HCOOH}$ is also readily produced in icy grain mantles (Tielens \& Hagen 1982; Allamandola \& Sandford 1990; Charnley 1995). Upon mantle evaporation, $\mathrm{HCOOH}$ will be released into the gas phase and can be detected at millimeter wavelengths (Liu et al. 2001, 2002). Ehrenfreund et al. (2001) have shown that formic acid is more abundant in the solid state, both in interstellar ices and cometary ices, than in the interstellar gas (ice/gas $\sim 10^{4}$ ); this point remains a puzzle and more laboratory work is necessary to clarify this question.

Sorrell (2001) has proposed the formation of the carboxyl group $(\mathrm{COOH})$ as a stable radical in the UV photolysis of $\mathrm{H}_{2} \mathrm{O} / \mathrm{CO}$ ice. Reactions in the gas phase and in the ice phase 
("ice-solvated phase") have been considered by Woon (2002) via quantum chemical modeling. Mendoza et al. (2004) have shown by theoretical calculations that $\mathrm{COOH}$ can also be generated on graphitic surfaces via adsorbate-adsorbate reactions.

The photodissociation of formic acid has been studied experimentally and theoretically in the vacuum ultraviolet (VUV) region (Su et al. 2000; Tabayashi et al. 1999; Schwell et al. 2002). However the results cannot explain the ice/gas ratio, since about $20 \%$ of formic acid survives the UV radiation. Despite some photo absorption studies in the $\mathrm{X}$-ray range (Ishii \& Hicthcock 1987; Prince et al. 2003) there are no studies focusing on the photodestruction of molecules by soft X-rays. The question about the relative abundances of formic acid in solid and gas phase is still open (Ehrenfreund et al. 2001). The present work aims to examine the photodissociation of formic acid by soft X-rays, at energies around the carbon $\mathrm{K}$ edge.

\section{Experimental}

The experimental set-up has been described in detail elsewhere (Lago et al. 2004 and de Souza et al. 2000). Briefly, the experiment was performed at the Brazilian Synchrotron Light Source (LNLS), Campinas, São Paulo, Brazil. Soft X-rays photons $\left(\sim 10^{12}\right.$ photons/s) from a toroidal grating monochromator (TGM) beamline (100-310 eV) perpendicularly intersect the effusive gaseous sample inside a high vacuum chamber. The base pressure in the vacuum chamber was in the $10^{-8}$ Torr range. During the experiment the chamber pressure is maintained below $10^{-5}$ Torr. The gas needle is kept at ground potential. The emergent photon beam is recorded by a light sensitive diode. The sample was commercially obtained from SigmaAldrich with purity better than $99.5 \%$. No further purification was used except for degassing the liquid sample by multiple freeze-pump-thaw cycles before admitting the vapor into the chamber. Conventional time-of-flight mass spectra were obtained using the correlation between one Photoelectron and a Photoion Coincidence (PEPICO). The ionized recoil fragments produced by the interaction with the photon beam are accelerated by a two-stage electric field and detected by two microchannel plate detectors in a chevron configuration after being mass-to-charge $(\mathrm{m} / \mathrm{q})$ analyzed by a time-of-flight mass spectrometer (TOF-MS). They produce up to three stop signals to a time-to-digital converter (TDC) started by the signal from one of the electrons accelerated in the opposite direction and recorded without energy analysis by two micro-channel plate detectors. Besides PEPICO spectra, other two kinds of coincidence mass spectra were obtained simultaneously (Pilling et al. 2005, in prep.): PE2PICO spectra (PhotoElectron Photoion Photoion Coincidence) and PE3PICO spectra (PhotoElectron Photoion Photoion Photoion Coincidence). Those spectra have ions coming from double and triple ionization process, respectively, that arrive coincidentally with a photoelectron. In both cases the multiple ionization is associated with the Auger process. Of all signals received by the detectors only about $10 \%$ come from PE2PICO and 1\% from PE3PICO spectra, reflecting that the majority contribution is indeed due to single event coincidence.
The pressure at the interaction region (volume defined by the gas beam and the photon beam intersection) was estimated to be $\sim 1$ Torr. At this pressure about $30 \%$ of $\mathrm{HCOOH}$ molecules are dimers (Halford 1942; Barton et al. 1969) but no obvious consequences of these clusters were seen in the detected spectra. The measurements were done at room temperature.

The first stage of the electric field $(708 \mathrm{~V} / \mathrm{cm})$ consists of a plate-grid system crossed by the light beam at the center. The TOF-MS was designed to have a maximized efficiency for ions with energies up to $30 \mathrm{eV}$ (Willey \& McLaren 1955). The secondary electrons produced in the ionization region are focused by an electrostatic lens polarizing the electron grid with $800 \mathrm{~V}$, designed to focus them at the center of the micro-channel plate detector. Negative ions may also be produced and detected, but the corresponding cross-sections are negligible. Our experimental setup does not have an electron energy analyzer to measure the photoelectron energies.

\section{Results and discussion}

Figure 1 shows a mass spectrum of the formic acid obtained at $300 \mathrm{eV}$ photon energy. Some general observations can be made. The $\mathrm{CO}^{+}$ion production is the most likely outcome (36-40\%) in the dissociation of the formic acid molecule in the energy range studied in the present work. This is followed by $\mathrm{CH}_{2}^{+}$(with a possible contribution of same $\mathrm{m} / \mathrm{q}$ ion $\mathrm{CO}^{++}$) $(18-27 \%), \mathrm{O}^{+}, \mathrm{HCO}^{+}\left(\right.$or $\left.\mathrm{COH}^{+}\right)$and $\mathrm{O}_{2}^{+}(\sim 14 \%)$ fragments, the latter coming from rearrangements. The $\mathrm{CH}_{2}^{+}+\mathrm{CO}^{++}$peak rises to very broad structures usually associated with a large intrinsic kinetic energy.

In Fig. 2 we show details of three regions of a mass spectrum of formic acid obtained in the energy range from 200 to $310 \mathrm{eV}$, around the $\mathrm{C} 1 \mathrm{~s}$ resonance ( $\left.1 \mathrm{~s} \rightarrow 2 \pi^{*}\right)$, exhibiting peak profiles of $\mathrm{H}^{+}, \mathrm{HCO}^{+}, \mathrm{COO}^{+}, \mathrm{COOH}^{+}$and $\mathrm{HCOOH}^{+}$ions. To clarify the mechanism leading to the molecular fragmentation, we observe that the mentioned ions appear in the mass spectra only above $275 \mathrm{eV}$ suggesting that the main contribution to the fragmentation processes comes from a resonant-type Auger decay, which takes place after the electron excitation to unoccupied Rydberg or antibonding orbitals, and might be responsible for the appearance of the $\mathrm{HCOOH}^{+}$parent ion. This kind of resonant-type Auger decay creates a hole in the valence orbital and its final state is identical to a direct valence photoionization. Below $275 \mathrm{eV}$, the hole can be in an inner valence orbital, favoring the full molecular fragmentation preferentially into the $\mathrm{CO}^{+}$plus neutrals or $\mathrm{CH}_{2}^{+}$plus neutrals.

The reactive ions $\mathrm{CO}^{+}$and $\mathrm{COH}^{+}$that are photodissociation product, were detected toward the H II region Monoceros R2 (Rizzo 2003) and in three photodissociation region, the reflection nebula NGC 7023, the Orion Bar and the planetary nebula NGC 7027 (Fuente et al. 2003). Usero et al. (2004) reported the first extragalactic detection of the reactive ion $\mathrm{COH}^{+}$in the circumnuclear disk of Active Galactic Nuclei, NGC 1068. They concluded that X-rays can heavily influence the physical conditions and chemical abundances of molecular gas and that the circumnuclear disk has become a giant X-ray Dominated Region (XDR). 


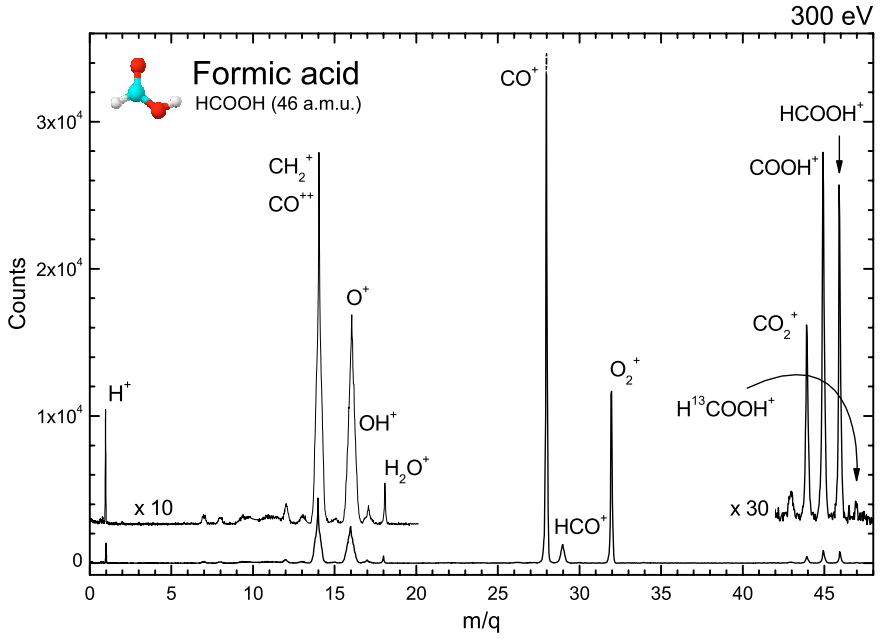

Fig. 1. Time-of-flight mass spectrum of $\mathrm{HCOOH}$ molecule recorded at $300 \mathrm{eV}$.

Another feature is the fate of the $\mathrm{H}^{+}, \mathrm{C}^{+}$and $\mathrm{CH}^{+}$ions below $280 \mathrm{eV}$ photon energy. One possible explanation for this behavior is the fact that dissociation occurs less often. It seems to be associated with the fate of the parent molecule. One possibility is that the molecule remains intact and neutral. Only radiative decay could result in uncharged molecules, but the radiative yields are expected to be very low in the present energy range. Corresponding to the increase in the $\mathrm{H}^{+}$, $\mathrm{C}^{+}$and $\mathrm{CH}^{+}$yields is a relative decrease in the relative yields of $\mathrm{CH}_{2}^{+}+\mathrm{CO}^{++}$, suggesting that the peak at $m / q=14$ is dominated by the $\mathrm{CH}_{2}^{+}$and not by the doubly charged $\mathrm{CO}^{++}$fragment. From the mass spectra one can observe an increase in the $\mathrm{H}^{+}$peak intensity due to the increase in the kinetic energy release as a function of the photon energy $(2.2 \mathrm{eV}$ at 280 and $290 \mathrm{eV}, 3.9$ at $300 \mathrm{eV}$ and 4.8 at $310 \mathrm{eV}$ ). Below $280 \mathrm{eV}$, the kinetic energy of the $\mathrm{H}^{+}$fragment is not enough to escape, being recaptured to form the $\mathrm{CH}_{2}^{+}$ion. As the photon energy increases, so does the kinetic energy of $\mathrm{H}^{+}$, increasing the probability of forming $\mathrm{H}^{+}$.

The doubly charged fragments $\mathrm{O}^{++}$and $\mathrm{CH}_{2}^{++}$are also observed from $275 \mathrm{eV}$ and above. The absence of more doubly ionized fragments in the PEPICO spectra indicates that the doubly ionized formic acid dissociates preferentially via charge separation. The dynamics of the doubly ionized formic acid molecule will be the subject of a future publication.

Figure 3 shows the partial ion yields (PIY) for the most significant outcomes in the dissociation of formic acid in the 200-310 eV photon energy range, the yields of $\mathrm{H}^{+}, \mathrm{HCO}^{+}$, $\mathrm{COOH}^{+}$and $\mathrm{HCOOH}^{+}$are also shown. A small dip can be seen in the fractions of $\mathrm{CO}^{+}$and $\mathrm{O}_{2}^{+}$at the $\mathrm{C} 1 \mathrm{~s}$ edge. Above $290 \mathrm{eV}$ this fraction shows a drop as a function of energy. The fraction of $\mathrm{CH}_{2}^{+}$fragments also drops around the $\mathrm{C}$ 1s edge while the fraction of the $\mathrm{C}^{+}, \mathrm{CH}^{+}, \mathrm{HCO}^{+}, \mathrm{OH}^{+}$and $\mathrm{H}^{+}$exhibit a gradual increase, being totally absent below $275 \mathrm{eV}$. This observation strongly indicates that $\mathrm{H}^{+}, \mathrm{C}^{+}, \mathrm{CH}^{+}, \mathrm{H}_{2} \mathrm{O}^{+}, \mathrm{OH}^{+}$, and $\mathrm{HCO}^{+}$ are only formed after the normal-type Auger decay (a small contribution of $\mathrm{HCO}^{+}$of roughly $0.3 \%$ is observed at $275 \mathrm{eV}$ ).

In Fig. 4 we show a comparison between partial ion yield in soft X-rays $(290 \mathrm{eV})$ obtained at LNLS with the one in UV (by
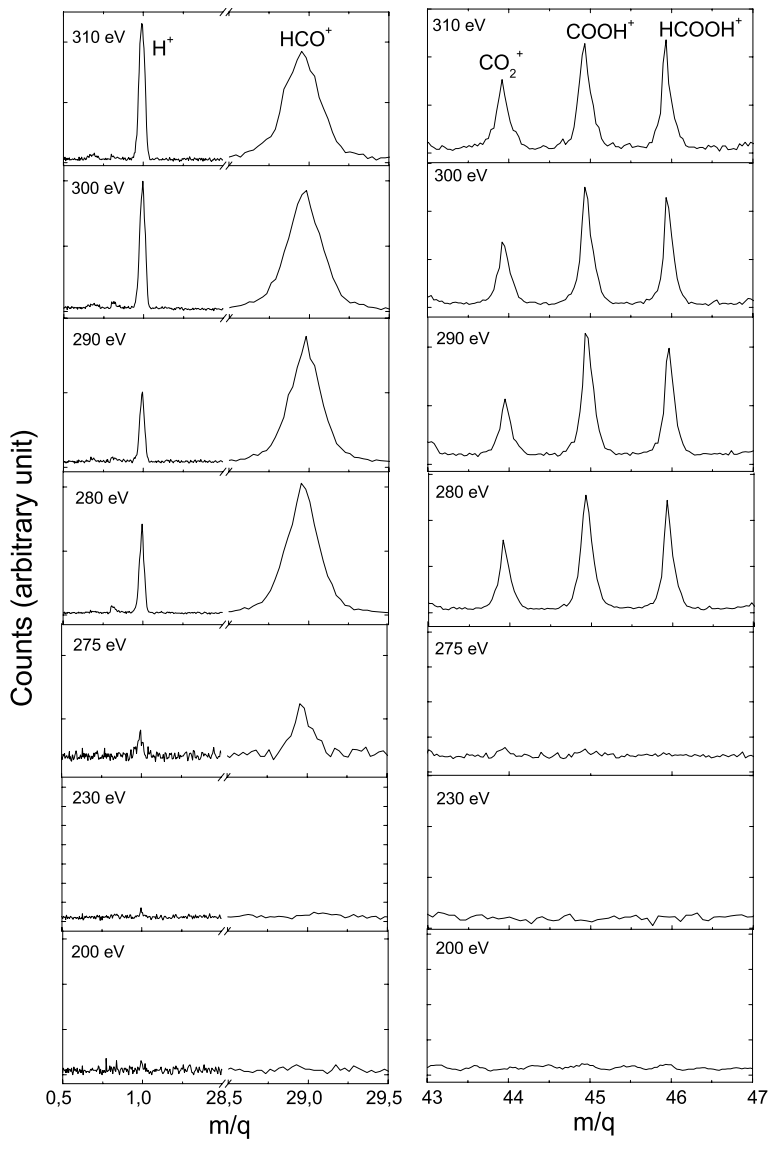

Fig. 2. Time-of-flight mass spectra of formic acid molecule showing details of $\mathrm{H}^{+}, \mathrm{HCO}^{+}$(left panel) and $\mathrm{CO}_{2}^{+}, \mathrm{COOH}^{+}$and $\mathrm{HCOOH}^{+}$ ions (right panel) for several photon energies.

$70 \mathrm{eV}$ electrons $)^{1}$ from NIST${ }^{2}$. The molecular ion $\mathrm{HCOOH}^{+}$ is more destroyed by soft X-rays than by a low UV field. The partial ion yields of several fragments also present differences in the X-ray field when compared with UV field, for example the large enhancement of $\mathrm{CO}^{+}$production by X-rays. The same is true for $\mathrm{O}^{+}, \mathrm{O}_{2}^{+}$and $\mathrm{CH}_{2}^{+}$fragments. The opposite behavior occurs with $\mathrm{HCO}^{+}$and $\mathrm{OH}^{+}$which seem to be more efficiently produced by UV photons, as has been previously reported (Suto et al 1988; Su et al. 2000; Schwel et al. 2002).

A comparison of partial ion yield results of the $\mathrm{HCOOH}$ and $\mathrm{CO}_{2}$ molecules measured at photon energies of 290 and $291 \mathrm{eV}$, respectively, can be seen in Fig. 5. The $\mathrm{CO}_{2}$ survives about $10 \%$ more than $\mathrm{HCOOH}$ and its main products by photodissociation are $\mathrm{O}^{+}$and $\mathrm{C}^{+}$at $40 \%$ and $27 \%$, respectively. Those ionic fragments represent only $13 \%$ and $1 \%$ of all fragments in the case of formic acid photodissociation. The $\mathrm{HCOOH}$ dissociation is two times more efficient for $\mathrm{CO}^{+}$production than $\mathrm{CO}_{2}$ dissociation.

From the observations described in the preceding paragraphs, we suggest that below the $\mathrm{C} 1 \mathrm{~s}$ edge, the fragmentation

${ }^{1}$ The effect of a $70 \mathrm{eV}$ electron is very similar to $21.21 \mathrm{eV}$ (He I Lamp) photons; in both the main ionization occurs in a valence shell (see discussion in Lago et al. 2004).

2 National Institute of Standards and Technology

http://webbook.nist.gov/chemistry/ 


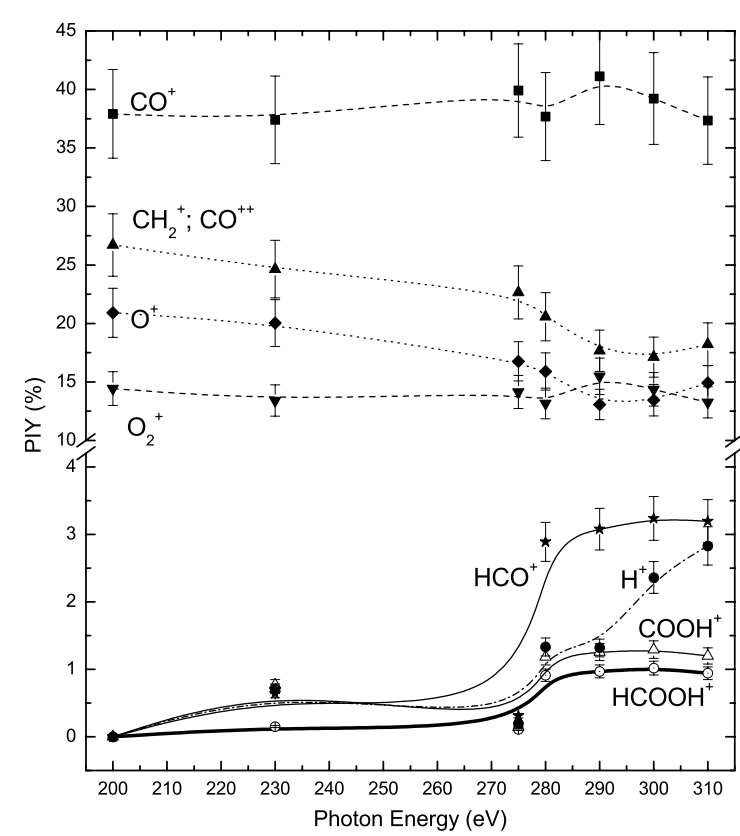

Fig. 3. Partial ion yield (PIY) of some PEPICO fragments of $\mathrm{HCOOH}$ molecule as a function of photon energy.

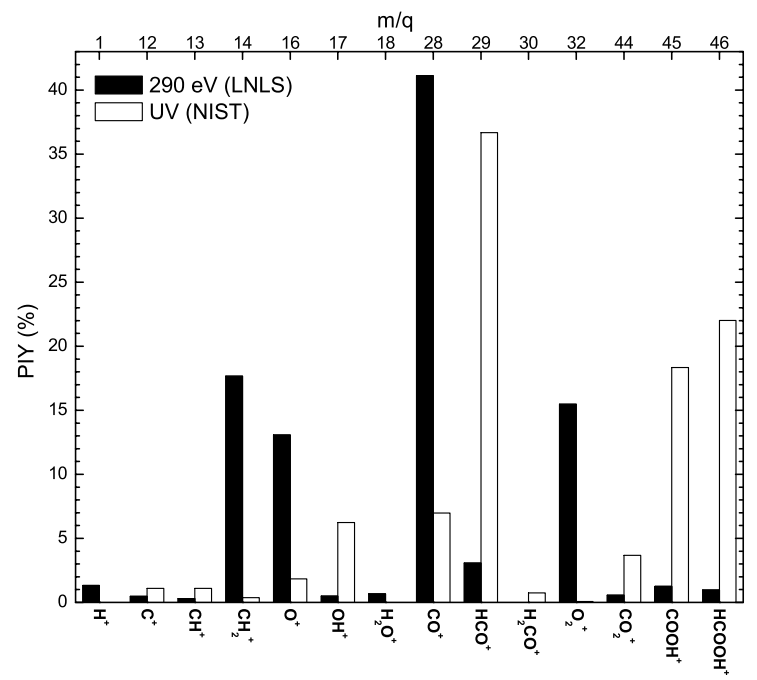

Fig. 4. Comparison between partial ion yield (PIY) in soft X ray field and UV field.

of the formic acid molecule is dominated by the pathways

$$
\begin{aligned}
\mathrm{HCOOH}+h v & \longrightarrow \mathrm{HCOOH}^{+}+\mathrm{e}^{-} \\
\mathrm{HCOOH}^{+} & \longrightarrow \mathrm{CO}^{+}+\mathrm{H}_{2} \mathrm{O}(\text { or } \mathrm{H}+\mathrm{OH}) \\
\mathrm{HCOOH}^{+} & \longrightarrow \mathrm{CH}_{2}^{+}+\mathrm{O}_{2}(\text { or } \mathrm{O}+\mathrm{O}) \\
\mathrm{HCOOH}^{+} & \longrightarrow \mathrm{O}^{+}+\mathrm{HCOH}(?) \\
\mathrm{HCOOH}^{+} & \longrightarrow \mathrm{O}_{2}^{+}+\mathrm{CH}_{2}(\text { or } \mathrm{CH}+\mathrm{H}) \\
\mathrm{HCOOH}^{+} & \longrightarrow \mathrm{HCO}^{+}+\mathrm{OH}(\text { or } \mathrm{O}+\mathrm{H})
\end{aligned}
$$

\subsection{Kinetic energy release (heating) of the ionic fragments}

Several authors have recently focused on the pathway of formation of biomolecules present in the star-forming region and other gaseous-dusty astronomical media (Largo 2004;

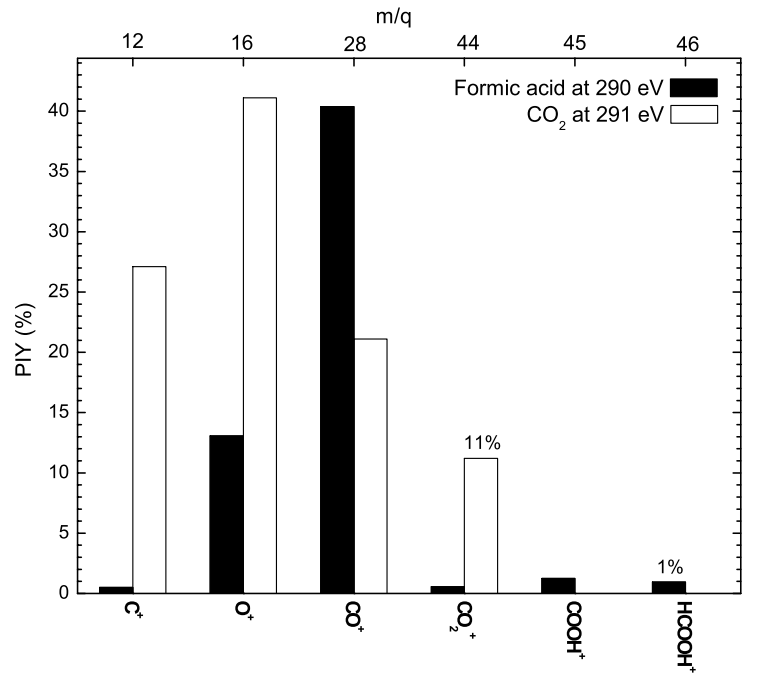

Fig. 5. Comparison between partial ion yield (PIY) of $\mathrm{HCOOH}$ (this work) and $\mathrm{CO}_{2}$ (Hitchcock et al. 1979).

Woon 2002, and references therein). Despite the success of ab initio theoretical calculations, the endothermic ion-molecule reactions have always been discounted, and only exothermic reactions have been accepted as a viable mechanism. However, with the knowledge of the kinetic energy (or at least with its value range) of some radical and ionic fragments, some endothermic ion-molecule reactions could be competitive and, in extreme situations, even become more efficient than those exothermic reactions.

We have determined the kinetic energy of all cationic fragments from the photodissociation of formic acid.

The present time-of flight spectrometer was designed to fulfil the Wiley-McLaren conditions for space focusing (Wiley \& McLaren 1955). Within the space focusing conditions, the observed broadening of peaks in spectra is mainly due to kinetic energy release of fragments. Considering that the electric field in the interaction region is uniform, we can determine the released energy in the fragmentation process $\left(U_{0}\right)$ from each peak width used by Simon et al. (1991), Hansen et al. (1998), Santos et al. (2001)

$U_{0}=\left(\frac{q E \Delta t}{2}\right)^{2} \frac{1}{2 m}$

where $q$ is the ion fragment charge, $E$ the electric field in interaction region, $m$ is the mass of fragment, and $\Delta t$ is the time peak width (FWHM) taken from PEPICO spectra. In order to test the above equation we have measured the argon mass spectrum under the same conditions. The kinetic energy value achieved for the $\mathrm{Ar}^{+}$ions is in agreement with the mean kinetic energy (3/2)KT obtained assuming Maxwell's distribution law. The calculated values for kinetic energy release $\left(U_{0}\right)$ for formic acid fragmentation is shown in Table 1.

We observe that the highest kinetic energy release was associated with the lightest fragment $\mathrm{H}^{+}(m / q=1)$, as expected. Extremely fast ionic fragments $\left(U_{0}>10 \mathrm{eV}\right)$, usually associated with dissociation of doubly or multiply-charged ions were also observed at high photon energies. These observations point to the important role of Auger process ion fragmentation 
Table 1. Relative intensities (partial ion yield - PIY) and kinetic energy $U_{0}$ release by fragments in the formic acid mass spectra, as a function of photon energy. Only fragments with intensity $>0.1 \%$ were tabulated. The estimated experimental error was $10 \%$

\begin{tabular}{|c|c|c|c|c|c|c|c|c|}
\hline \multicolumn{2}{|r|}{ Fragments } & \multicolumn{7}{|c|}{ PIY $(\%) / U_{0}(\mathrm{eV})$} \\
\hline$m / q$ & Attribution & $200 \mathrm{eV}$ & $230 \mathrm{eV}$ & $275 \mathrm{eV}$ & $280 \mathrm{eV}$ & $290 \mathrm{eV}$ & $300 \mathrm{eV}$ & $310 \mathrm{eV}$ \\
\hline 1 & $\mathrm{H}^{+}$ & - & $0.71 / 0.24$ & $0.19 / 0.24$ & $1.33 / 2.17$ & $1.32 / 2.17$ & $2.36 / 2.87$ & $2.83 / 4.80$ \\
\hline 7 & $\mathrm{CH}_{2}^{++} ; \mathrm{CO}^{++++} ?$ & - & $1.57 / 30.5$ & $0.86 / 11.0$ & $0.72 / 13.8$ & $0.47 / 8.84$ & $0.52 / 9.97$ & $0.64 / 10.0$ \\
\hline 8 & $\mathrm{O}^{++}$ & - & - & $0.65 / 2.45$ & $0.57 / 12.0$ & $0.30 / 10.9$ & $0.38 / 8.71$ & $0.49 / 2.45$ \\
\hline 9.3 & $\mathrm{CO}^{+++} ?$ & - & - & $1.21 / 52.0$ & $0.91 / 87.8$ & $0.83 / 67.5$ & $0.91 / 33.5$ & $0.95 / 45.8$ \\
\hline 12 & $\mathrm{C}^{+}$ & - & - & $0.26 / 1.28$ & $0.56 / 0.50$ & $0.49 / 0.40$ & $0.93 / 0.98$ & $1.19 / 0.98$ \\
\hline 13 & $\mathrm{CH}^{+}$ & - & - & - & $0.30 / 1.18$ & $0.30 / 1.04$ & $0.49 / 1.04$ & $0.47 / 0.16$ \\
\hline 14 & $\mathrm{CH}_{2}^{+} ; \mathrm{CO}^{++}$ & $26.7 / 0.07$ & $24.6 / 0.11$ & $22.6 / 0.72$ & $20.6 / 0.72$ & $17.7 / 0.61$ & $17.1 / 0.43$ & $18.2 / 0.96$ \\
\hline 16 & $\mathrm{O}^{+}$ & $20.9 / 0.30$ & $20.0 / 1.99$ & $16.8 / 1.99$ & $15.9 / 1.65$ & $13.1 / 0.96$ & $13.4 / 1.22$ & $14.9 / 1.50$ \\
\hline 17 & $\mathrm{OH}^{+}$ & - & - & - & $0.45 / 0.06$ & $0.49 / 0.17$ & $0.59 / 0.13$ & $0.75 / 0.17$ \\
\hline 18 & $\mathrm{H}_{2} \mathrm{O}^{+}$ & - & $0.66 / 0.12$ & $0.25 / 0.05$ & $0.55 / 0.05$ & $0.67 / 0.03$ & $0.64 / 0.03$ & $0.60 / 0.05$ \\
\hline 28 & $\mathrm{CO}^{+}$ & $37.9 / 0.02$ & $37.4 / 0.02$ & $39.9 / 0.02$ & $37.7 / 0.02$ & $41.1 / 0.02$ & $39.2 / 0.02$ & $37.3 / 0.03$ \\
\hline 29 & $\mathrm{HCO}^{+}$ & - & $0.64 / 0.10$ & $0.31 / 0.03$ & $2.89 / 0.13$ & $3.08 / 0.13$ & $3.24 / 0.17$ & $3.19 / 0.16$ \\
\hline 32 & $\mathrm{O}_{2}^{+}$ & $14.4 / 0.03$ & $13.4 / 0.03$ & $14.1 / 0.03$ & $13.2 / 0.02$ & $15.5 / 0.02$ & 14.4 / 0.02 & $13.3 / 0.01$ \\
\hline 44 & $\mathrm{CO}_{2}^{+}$ & - & - & $0.16 / 0.03$ & $0.63 / 0.02$ & $0.56 / 0.02$ & $0.72 / 0.02$ & $0.75 / 0.03$ \\
\hline 45 & $\mathrm{COOH}^{+}$ & - & $0.76 / 0.01$ & $0.14 / 0.16$ & $1.18 / 0.03$ & $1.26 / 0.03$ & $1.29 / 0.02$ & $1.19 / 0.03$ \\
\hline 46 & $\mathrm{HCOOH}^{+}$ & - & $0.14 / 0.11$ & $0.11 / 0.06$ & $0.91 / 0.01$ & $0.97 / 0.01$ & $1.02 / 0.02$ & $0.94 / 0.01$ \\
\hline
\end{tabular}

of core-ionized polyatomic molecules. The Coulomb explosion associated with the Auger process should explain the increase in kinetic energy of the ionic fragments, reflected in the increasing broadening of several fragments. This broadening observed in simple coincidence spectra (PEPICO) and its consequence on the shape of peaks in mass spectra has been discussed by Simon et al. (1993).

Averaging the kinetic energy release $U_{0}$ of each fragment by the fragmentation PIY we have found the mean kinetic energy release $\left\langle U_{0}\right\rangle_{\varepsilon}$ due to a photon of energy $\varepsilon$, considering all fragments in single coincidence spectra.

$\left\langle U_{0}\right\rangle_{\varepsilon}=\frac{\sum_{f} \mathrm{PIY}_{f} \times U_{0_{f}}}{100}$.

Assuming Maxwell's distribution of velocities we calculated the mean kinetic temperature of all fragments in the interaction region. This temperature can be seen in Fig. 6 as a function of photon energy. Photons with energies close to the $\mathrm{C} 1 \mathrm{~s}$ threshold photoionization energy seem to produce faster ionized fragments which can been associated with some kind of resonance in molecule. Averaging over all photon energies we can estimate the total heating $\mathrm{H}$ of ionic fragmentation release above room temperature by

$H=\left\langle T_{0}\right\rangle-T_{\text {room }}=\frac{2}{3} \frac{1}{k} \frac{\sum_{\varepsilon}\left\langle U_{0}\right\rangle_{\varepsilon}}{n}-290 \mathrm{~K}$

where $\left\langle T_{0}\right\rangle$ is the mean kinetic temperature of all cationic fragments averaged in all photon energies, $k$ is the Boltzmann constant $\left(8.61 \times 10^{-5} \mathrm{eV} \mathrm{K}^{-1}, \varepsilon\right.$ is the photon energy in $\mathrm{eV}$ and $\mathrm{n}$ is the total number of spectra in our experimental data.

We have found the value $H \sim 7000 \mathrm{~K}(\sim 0.9 \mathrm{eV})$. This result shows that photoionization by an X-ray field can increase the local temperature since several fragments could reach high values of kinetic energy.

As the C 1s ionizing potential of formic acid is $295.8 \mathrm{eV}$ (Prince et al. 2003) and using soft X-ray photons, the heating $H$ produced by ejected inner shell photoelectrons is about 5-15 times greater than that caused by ionic fragments.

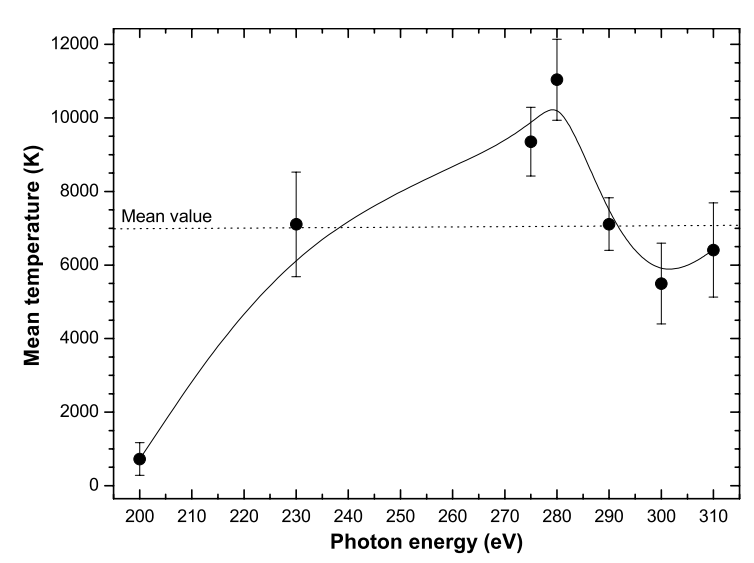

Fig. 6. Mean Kinetic temperature of ionic fragments of formic acid molecule as a function of photon energy.

The study of the decay of core-excited molecules provides information about the bonding or antibonding nature of the molecular orbitals. Generally, the final electronic states of a core excited molecule are unknown due to the fact that the densities of the states are very high, and the bond distances and angles differ from their ground state configuration. The surface potentials of the ionic states are extremely repulsive. For core excited molecules which dissociate in one charged and one or more neutral fragments, the dissociation is primarily controlled by chemical (non-Coulomb) forces originating from the residual valence electrons of the system (Nenner \& Morin 1996). From Table 1, one can see that the mean kinetic energy release $U_{0}$ of some formic acid fragments increases as the photon energy approximates the $\mathrm{C} 1 \mathrm{~s}$ edge $(288 \mathrm{eV})$. This enhancement is due to the repulsive character of the $\sigma^{*}\left(\pi^{*}\right)$ resonance.

The maximum possible kinetic energy release available to a molecular fragment is given by the difference between the deposited photon energy and the appearance energy of the fragment. Lorquet (2000) has shown that the relationship between the average kinetic energy release $U_{0}$ and the internal energy 
measured in excess of the dissociation threshold, $\Delta E$, is not linear. It contains information about the density of vibrationalrotational states. The mean kinetic energy release is found to increase as $\Delta E^{1 / 2}$. This behavior can be seen in Fig. 6.

\subsection{Absolute photoionization and photodissociation cross sections}

Sorrell (2001) proposed a theoretical model for the chemical manufacture of interstellar aminoacids and sugars, assuming that those biomolecules are formed inside the bulk interior of icy grain mantles photoprocessed by starlight (ultraviolet and soft X-rays photons). His model chemistry is based on radicalradical reactions followed by chemical explosions of a processed mantle that ejects large amounts of organic dust into the ambient gaseous medium. The density number of a given biomolecule in a steady state regime of creation and destruction inside a gaseous-dusty cloud is given by

$N_{\mathrm{Mol}}=\frac{\dot{N}_{\mathrm{Mol}} n_{\mathrm{d}}}{\left\langle\sigma_{\mathrm{ph}-\mathrm{d}}\right\rangle I_{0}}$

where $\dot{N}_{\text {Mol }}$ is the molecule ejection rate which depends mainly on the molecule mass and the properties of grains and the cloud (see Eq. (21) of Sorrell 2001), $n_{\mathrm{d}}$ is the dust space density and $I_{0}$ is the number flux of ionizing photons (photons $\mathrm{cm}^{-2} \mathrm{~s}^{-1}$ ) inside the cloud and $\left\langle\sigma_{\mathrm{ph}-\mathrm{d}}\right\rangle$ is the averaged photodissociation cross section in wavelength range of the photon flux density.

As mentioned by Sorrell (2001), the main uncertainty of this model equilibrium abundance cames from the uncertainty of $\sigma_{\mathrm{ph}-\mathrm{d}}$ value. Therefore the precise determination of $\sigma_{\mathrm{ph}-\mathrm{d}}$ of biomolecules is very important to estimate the molecular abundance of those molecules in the interstellar medium. Moreover, knowing the photon dose $\phi$ and $\sigma_{\mathrm{ph}-\mathrm{d}}$ value we can also determine the half-life of given molecule as discussed by Bernstein et al. (2004).

The photodissociation rates, $R$, of a molecule dissociated by the interstellar radiation field $I_{\varepsilon}$ in the energy range, $\varepsilon_{2}-\varepsilon_{1}$, is given by

$R=\int_{\varepsilon_{1}}^{\varepsilon_{2}} \sigma_{\mathrm{ph}-\mathrm{d}}(\varepsilon) I_{0}(\varepsilon) \mathrm{d} \varepsilon$

where $\sigma_{\mathrm{ph}-\mathrm{d}}(\varepsilon)$ is the photo-dissociation cross section as a function of photon energy $\left(\mathrm{cm}^{2}\right), I_{0}(\varepsilon)$ is the photon flux as a function of energy (photons $\mathrm{cm}^{-2} \mathrm{eV}^{-1} \mathrm{~s}^{-1}$ ) (see discussion in Cottin et al. 2003; Lee 1984).

From Eq. (5) we can also derive the half-life time, $t_{1 / 2}$, of the molecule as

$t_{1 / 2}=\frac{\ln 2}{\int_{\varepsilon_{1}}^{\varepsilon_{2}} \sigma_{\mathrm{ph}-\mathrm{d}}(\varepsilon) I_{0}(\varepsilon) \mathrm{d} \varepsilon}$

which does not depend on the molecular number density.

In order to put our data on an absolute scale, we have summed all the contributions of all cationic fragments detected and normalized them to the photoabsorption cross sections measured by Ishii \& Hitchcock (1987).

After a subtraction of a linear background and false coincidences coming from aborted double and triple ionization (see
Simon et al. 1991), the integrated true coincidence signal $I_{f}^{q+}$ for a given fragment $f$ with charge $q$, at constant photon flux and lower target pressures is given by

$I_{f}^{q+}=\epsilon_{e_{q^{+}}} \epsilon_{i} I_{0} n t_{\exp } \sigma_{f}^{q+}=\epsilon_{e_{q^{+}}} \epsilon_{i} K \sigma_{f}^{q+}$

where $I_{0}$ is the total photon number that reaches the molecular beam (photons $\mathrm{s}^{-1}$ ), $n$ is the number of target scattering centers per area (as function of the collision region pressure), $t_{\exp }$ is the experiment acquisition time, $\sigma^{q+}$ is the ionization cross section $^{3} \epsilon_{i}$ and $\epsilon_{e_{q+}}$ are the ion and the electron ${ }^{4}$ detection efficiencies, respectively, where once again $q$ designates the number of ejected electrons. The adopted efficiency values were $\epsilon_{i}=0.23$; $\epsilon_{e_{1+}}=0.04 ; \epsilon_{e_{2+}}=0.54 ; \epsilon_{e_{3+}}=0.78$ (Cardoso 2001).

Since the absolute target density $n$ as well as the incident number of photons $I_{0}$ have not been determined absolutely, we take $K \equiv I_{0} n t_{\text {exp }}$ as a constant (in $\mathrm{cm}^{-2}$ ) to determined for each given energy photon.

The total integrated coincidence signal for a given photon energy and final charge state $q+$ is given by

$I^{q+}=\sum_{f} I_{f}^{q+}=\epsilon_{e_{q^{+}}} \epsilon_{i}^{q} K \sigma^{q+}$.

Analogously, the total ionization cross section is given by

$\sigma^{q+}=\sum_{f} \sigma_{f}^{q+}=\frac{1}{K} \frac{I^{q+}}{\epsilon_{e^{++}} \epsilon_{i}^{q}}$.

Assuming a negligible fluorescence yield (due to the low carbon atomic number (Chen et al. 1981)) and anionic fragments production, we adopted that all absorbed photon lead to cationic ionizing process, therefore we can write

$\sigma_{a b s} \equiv \sum_{q=1}^{3} \sigma^{q+}=\frac{1}{K} \sum_{q=1}^{3} \frac{I^{q^{+}}}{\epsilon_{e_{q^{+}}} \epsilon_{i}^{q}}$

or yet

$K=\frac{\sum_{q=1}^{3} \frac{I^{q+}}{\epsilon_{e_{++}} \epsilon_{i}^{q}}}{\sigma_{\mathrm{abs}}}$

Therefore, we can calculate the cross section for single ionized $(q=1)$ fragments:

$\sigma^{+}=\frac{I^{+}}{\epsilon_{e_{+}} \epsilon_{i}} \frac{1}{K}$.

The non-dissociative single ionization (photoionization) cross section $\sigma_{\mathrm{ph}-\mathrm{i}}$ and the dissociative single ionization (photodissociation) cross section $\sigma_{\mathrm{ph}-\mathrm{d}}$ of formic acid can be determined by

$\sigma_{\mathrm{ph}-\mathrm{i}}=\sigma^{+} \frac{\mathrm{PIY}_{\mathrm{HCOOH}^{+}}}{100}$.

${ }^{3} \sigma^{+}, \sigma^{2+}$ and $\sigma^{3+}$ are the single, double and triple ionization cross section obtained form PEPICO, PE2PICO and PE3PICO spectra, respectively.

4 The $\epsilon_{e_{2+}}$ is the efficiency of detecting at least one of two electrons released by double ionization processes in PE2PICO spectra and $\epsilon_{e_{3+}}$ is the efficiency of detecting at least one of three electrons released by triple ionization processes in PE3PICO spectra. 


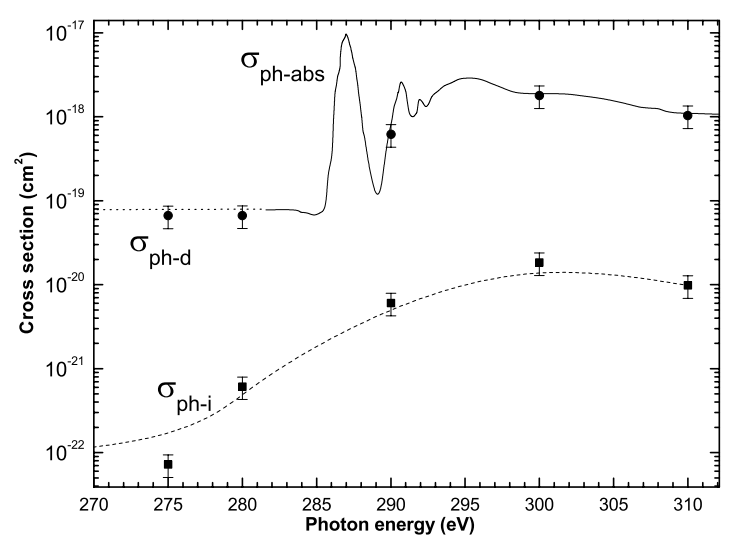

Fig. 7. Non-dissociative single ionization (photoionization) cross section $\left(\sigma_{\mathrm{ph}-\mathrm{i}}\right)$ and dissociative ionization (photodissociation) cross section $\left(\sigma_{\mathrm{ph}-\mathrm{d}}\right)$ of formic acid as a function of photon energy. The photoabsorption cross section $\sigma_{\mathrm{ph}-\mathrm{abs}}$ taken from Ishii \& Hitchcock (1987) is also shown.

Table 2. Values of non-dissociative single ionization (photoionization) cross section $\left(\sigma_{\mathrm{ph}-\mathrm{i}}\right)$ and dissociative ionization (photodissociation) cross section $\left(\sigma_{\mathrm{ph}-\mathrm{d}}\right)$ of formic acid as a function of photon energy. The estimated experimental error was 30\%. The photoabsorption cross section $\sigma_{\text {ph-abs }}$ from Ishii \& Hitchcock (1987).

\begin{tabular}{lccc}
\hline \hline \multirow{2}{*}{$\begin{array}{l}\text { Photon } \\
\text { energy }(\mathrm{eV})\end{array}$} & \multicolumn{3}{c}{ Cross sections $\left(\mathrm{cm}^{2}\right)$} \\
\cline { 2 - 4 } 275 & $\sigma_{\mathrm{ph}-\mathrm{d}}$ & $\sigma_{\mathrm{ph}-\mathrm{i}}$ & $\sigma_{\mathrm{ph}-\mathrm{abs}}$ \\
\hline 280 & $6.61 \times 10^{-20}$ & $7.21 \times 10^{-23}$ & $7.00^{a} \times 10^{-20}$ \\
290 & $6.64 \times 10^{-20}$ & $6.12 \times 10^{-22}$ & $7.00^{a} \times 10^{-20}$ \\
300 & $6.20 \times 10^{-19}$ & $6.06 \times 10^{-21}$ & $6.42 \times 10^{-19}$ \\
310 & $1.78 \times 10^{-18}$ & $1.83 \times 10^{-20}$ & $1.88 \times 10^{-18}$ \\
\hline${ }^{a}$ Ext & $1.03 \times 10^{-18}$ & $9.83 \times 10^{-21}$ & $1.10 \times 10^{-18}$ \\
\hline
\end{tabular}

${ }^{a}$ Extrapolated data (see Fig. 7).

In the same way the photodissociation cross section of formic acid can be determined by

$\sigma_{\mathrm{ph}-\mathrm{d}}=\sigma^{+}\left(1-\frac{\mathrm{PIY}_{\mathrm{HCOOH}^{+}}}{100}\right)$.

Both cross sections can be seen in Fig. 7 as a function of photon energy. The absolute absorption cross section of formic acid (Ishii \& Hitchcoch 1987) is also shown for comparison. Those values are also shown in Table 2.

The cross sections determined here should be useful in discussions of interstellar chemistry.

\section{Summary and conclusions}

The goal of this work is to experimentally study ionization and photodissociation processes of a glycine precursor molecule, $\mathrm{HCOOH}$ (formic acid). The measurements were taken at the Brazilian Synchrotron Light Laboratory (LNLS), employing soft X-ray photons from a toroidal grating monochromator TGM) beamline (200-310 eV). The experimental setup consists of a high vacuum chamber with a time-of-flight mass spectrometer TOF-MS. Mass spectra were obtained using PhotoElectron PhotoIon Coincidence (PEPICO) technique. Kinetic energy distributions and abundances for each ionic fragment were obtained from the analysis of the corresponding peak shapes in the mass spectra.

We have shown that the $\mathrm{X}$-ray field interactions with formic acid release a considerable number of energetic fragments, some of then with high kinetic energy (ex. $\left.\mathrm{H}^{+}, \mathrm{CH}^{+}, \mathrm{O}^{+}\right)$and some with extreme high kinetic energy $\left(\mathrm{CO}^{++}, \mathrm{CO}^{++}, \mathrm{O}^{++}\right)$. Those energetic fragments promote an increase in the local temperature of a region. An extension of this scenario to interstellar medium conditions could justify (or promote) some endothermic ion-molecule reactions and become important in elucidating some pathways of formation of complex molecules (Largo et al. 2004).

Dissociative and non-dissociative photoionization cross sections were also determined. Due to the high photodissociation cross section of formic acid it is possible that in PDR regions, just after molecule evaporation from the grain surface, it is almost destroyed by soft X-rays, justifying the observed low abundance of $\mathrm{HCOOH}$ in the gaseous phase. This result could indicate that the preferential path for the glycine formation via formic acid may be through ice.

Acknowledgements. The authors would like to thank the staff of the Brazilian Synchrotron Facility (LNLS) for their valuable help during course of the experiments. We are particularly grateful to Professor G. G. B. de Souza and Professor A. N. de Brito for the use of Timeof-Flight Mass Spectrometer. This work is supported by LNLS, CNPq and FAPERJ.

\section{References}

Allamandola, L. J., \& Sandford, S. A. 1990, NASA Conf. Pub., 3061, 113.

Barton, J. R., \& Hsu, C. C. 1969, J. Chem. Eng. Data, 14, 184

Bernstein, M. P., Ashbourn, S. F. M., Sandford, S. A., \& Allamandola, L. J. 2004, ApJ, 601, 365

Bottinelli, S., Ceccarelli, C., Lefloch, B., et al. 2004, ApJ, 615, 354

Cazaux, S., Tielens, A. G. G. M., Ceccarelli, C., et al. 2003, ApJ, 593, L51

Cardoso, E. S. 2001, MSc thesis, IF/UNICAMP

Charnley, S. B. 1995, Ap\&SS, 224, 251

Chen, M. H., Crasemann, B., \& Mark, H. 1981, Phys. Rev. A, 24, 177

Cottin, H., Moore, M. H., \& Bénilan, Y. 2003, ApJ, 590, 874

Crovisier, J., \& Bockelée-Morvan, D. 1999, Space Scien. Rev., 90, 19

de Souza, G. G. B., Santos, A. C. F., Rocco, M. L. M., et al. 2000, Quim. Nova, 24, 311

Ehrenfreund, P., \& Schutte, W. A. 2000, Adv. Space Res., 25, 2177

Ehrenfreund, P., D'Hendecourt, L., Charnley, S., \& Ruiterkamp, R. 2001, JGR, 106, 33291

Fuente, A., Rodriguez-Franco, A., Garcia-Burillo, S., Martin-Pintado, J., \& Black, J. H. 2003, A\&A, 406, 899

Goicoechea, J. R., Rodriguez-Fernandez, N. J., \& Cernicharo J. 2004, ApJ, 600, 214

Halford, J. O. 1942, J. Chem. Phys., 10, 582

Hansen, D. L., et al. 1998, Phys. Rev. A, 58, 5

Hitchcock, A. P., Brion, C. E., \& van der Wiel, M. J. 1979, Chem. Phys. Let. 66, 213

Ishii, I., \& Hitchcock, A. P. 1987, J. Chem. Phys. 2, 830

Jaffe, D. J., Becklin, E. E., \& Hildebrand, R. H. 1984, ApJ, 276, L51

Kuan, Y. J., Mehringer, D. M., \& Snyder L. E. 1996, ApJ, 459, 619 
Kuan, Y.-J., Charnley, S. B., Huang, H.-C., Tseng, W.-L., \& Kisiel, Z. 2003, ApJ, 593, Issue 2, 848

Kuan, Y. J., Charnley, S. B., Huang, H. C., et al. 2004, Adv. Space Res., 33, 31

Koyama, K., Hamaguchi, K., Ueno, S., Kobayashi, N., \& Feigelson E. D. 1996, PASJ, 48, L87

Lago, A. F., Santos, A. C. F., \& de Souza, G. G. B. 2004, J. Chem. Phys., 120, 9547

Largo, A., Redondo, P., \& Barrientos C. 2004, Int. J. Quant. Chem., 98, 355

Lee, L. C. 1984, ApJ, 282, 172

Liu, S. Y., Mehringer, D. M., \& Snyder, L. E. 2001, ApJ, 552, 654

Liu, S. Y., Girard, J. M., Remijan, A., \& Snyder, L. E. 2002, ApJ, 576, 255

Lorquet, J. C. 2000, J. Phys. Chem. A, 104, 5422

Mendoza, C., Ruette, F., Martorell, G., \& Rodrígez, L. S. 2004, ApJ, 601, L59

Nanda Kumar, M. S., Kamath, U. S., \& Davis, C. J., 2004 MNRAS, $353,4,1025$

Nenner, I., \& Morin, P. 1996, VUV and Soft X-Ray Photoinization, ed. Uwe Becker and D. A. Shirley (New York: Plenum Press)

Ohishi, M., Irvine, W. M., \& Kaifu, N. 1992, Astrochemistry of Cosmic Phenomena, ed. P. D. Singh (Dordrecht: Kluwer Academic Publishers), 171

Prince, K. C., Richter, R., Simone, M., Alagia, M., \& Coreno, M. 2003, J. Phys. Chem. A, 107, 1955

Rizzo, J. R., Fuente, A., Rodríguez-Franco, A., \& García-Burillo, S. 2003, ApJ, 597, L153

Santos, A. C. F., Lucas, C. A., \& de Souza, G. G. B. 2001, J. Electron Spectrosc. Relat. Phenom., 114, 115

Santos, A. C. F., Lucas, C. A., \& de Souza, G. G. B. 2002, Chem. Phys., 282, 315
Schwell, M., Dulieu, F., Jochims, H. W., et al. 2002 J. Phys. Chem A, 106(45), 10908

Schulz, N. S., Canizares, C., Huenemoerder, D., et al. 2001, ApJ, 549, 441

Simon, M., LeBrun, T., Morin, P., Lavolée, M., \& Maréchal, J. 1991, Nucl. Instrum. Methods B, 62, 167

Simon, M., LeBrun, T., Martins, R., et al. 1993, J. Phys. Chem., 97, 5228

Sorrell, W. H. 2001, ApJ, 555, L129

Su, H., Yong, H., \& Fanao, K. 2000, J. Chem. Phys., 113, 1891

Suto, M., Wang, X., \& Lee, L. C. 1988, J. Phys. Chem., 92, 3764

Sutton, E. C., Blake, G. A., Masson, C. R., \& Phillips, T. G. 1985, ApJS, 58, 341

Sjogren, B, de Brito, A., Correia, N., et al. 1992, J. Electron Spectrosc. Relat. Phenom., 59, 161

Tabayashi, K., Aoyama, J.-I., Matsui, M., Hino, T., \& Saito, K. 1999, J. Chem. Phys., 110, 9547

Takagi, S., Murakami, H. \& Koyama, K. 2002, The Proc. IAU 8th Asian-Pacific Regional Meeting, the Astronomical Society of Japan, 215

Tielens, A. G. G., \& Hagen, W. 1982, A\&A, 114, 225

Turner, B. E. 1991, ApJS, 76, 617

Wiley, W. E., \& McLaren, I. W. 1955, Rev. Sci. Instrum., 26, 1150

Woon, D. E. 2002, ApJ, 571, L17

Wright, M. C. H., Plambeck, R. L., \& Wilner, D. J. 1996, ApJ, 469, 216

Usero, A., García-Burillo, S., Fuente, A., Martín-Pintado, J., \& Rodríguez-Fernández, N. J. 2004, A\&A, 419, 897

Zombeck, M. V. 1990, Handbook of space astronomy \& Astrophysics, 2nd Edition (Cambridge University Press B)

Zuckermann, B., Ball, J. A., \& Gottlieb, A. C. 1971, ApJ, 163, L41 